Egor Lykov"

ETH Zürich
UDK 323.15(470.34=112.2):001.891

DOI: $10.4312 /$ linguistica.60.2.325-334

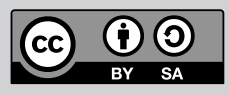

\title{
DIE WOLGADEUTSCHE KULTUR UND LITERATUR - FORSCHUNGSFELDER, -PROBLEME UND -DESIDERATA
}

\section{EINLEITUNG}

Die Erforschung von Kultur und Geschichte der Wolgadeutschen hat eine lange, tief im 19. Jahrhundert verwurzelte Geschichte. Am Anfang kam das Forschungsinteresse von den Wolgadeutschen selbst und hatte in erster Linie mit der Heimatkunde (Klaus 2010) und ferner mit dem aufkommenden Nationalismus im späten Russischen Reich zu tun (Kappeler 1996). Mit der Gründung der Autonomen Sozialistischen Sowjetrepublik der Wolgadeutschen (ASSRdWD), die von Historikerinnen und Historikern zum Höhepunkt der politischen Entwicklung der Wolgadeutschen erhoben wurde, verstärkte sich das Forschungsinteresse an der Kultur und Geschichte der Wolgadeutschen, während nach ihrer im Herbst 1941 erfolgten Zwangsumsiedlung in weit entlegene Gebiete der Sowjetunion kaum Forschungen auf diesem Gebiet betrieben wurden. Trotz vorhandener exemplarischer Studien aus der Sowjetunion nach 1945 kann von keiner etablierten Forschungsrichtung gesprochen werden (vgl. Smirnova 1992: 337-341). Das steigende Forschungsinteresse in den 1990er Jahren hing mit der „Wiederentdeckung“ der „deutschen Nation“" in Russland und massiven Einwanderung der Russlanddeutschen in die Bundesrepublik Deutschland zusammen, sodass die Forschungen zu Wolgadeutschen in der Regel einen zeithistorischen Fokus haben und nicht zuletzt auf Verifizierung der deutschen Abstammung einzelner Familien und Integration der Spätaussiedlerinnen und Spätaussiedler in der BRD abzielen (Boeckh: 252). Obwohl die Forschungen zur Geschichte und Kultur der Deutschen in Russland und in der Sowjetunion lange Zeit verpönt waren, handelt es sich hierbei um einen außerordentlich umfangreichen Fundus an wissenschaftlichen Arbeiten, sodass seine Behandlung den Rahmen dieses Artikels sprengen würde. Dennoch wird im vorliegenden Beitrag der Versuch unternommen, aktuelle Tendenzen der Wolgadeutschenforschung zu reflektieren und - von unveröffentlichten und zum Teil wenig benutzten Archivquellen ausgehend - neue Fragestellungen zu den Wolgadeutschen zu entwickeln. Der Beitrag soll in erster Linie als Anregung zur thematischen Weiterentwicklung der sog. Volga German Studies als eines interdisziplinären Forschungsfelds gelesen werden, wobei die relevantesten Forschungsergebnisse der letzten zwanzig Jahre in die Untersuchung einbezogen werden.

Die deutsche(n) Minderheit(en) in Russland hat/haben mehrere Namen, die den Eklektizismus dieser hybriden Identität(en) widerspiegeln: Wolgadeutsche, Russlanddeutsche, Deutschrussen (dt.); nemcy Povolžja, rossijskie nemcy, russkie nemcy (russ.); Russian Germans, German Russians, Germans from/of Russia (eng.), alemanes

egor.lykov@gta.arch.ethz.ch 
del Volga (span.) etc. (vgl. Schmaltz 2018: 41-72). ${ }^{1}$ Während Deutsche im Russischen Reich in mehr oder weniger geschlossenen einzelnen Sprachinseln an der Wolga, um St. Petersburg und Moskau herum, in Bessarabien, in der Ukraine, auf der Krim und seit 1911 auch im Bezirk Omsk und am Altai in Sibirien lebten, haben die massive Auswanderung der Deutschen aus dem Russischen Reich (1870-1914) und die dramatischen Ereignisse der russischen Revolution, des Bürgerkrieges und des Stalinschen Terrors sowie die bis dato andauernde Repatriierung in die BRD nach dem Zusammenbruch der Sowjetunion Deutsche aus Russland über die ganze Welt verstreut, woraus sich multiple Identitäten ergaben. Somit stellten Deutsche aus Russland nie eine homogene soziale Gruppe dar (vgl. Hilbrenner 2018: 31-34). Im Folgenden wird der Blick auf Wolgadeutsche im engeren Sinne gerichtet, die, dem räumlichen Prinzip folgend, als deutschsprechende autochthone Personen der Gouvernements Saratov und Samara im Russischen Reich und später in der ASSRdWD aufgefasst werden.

\section{FORSCHUNGSFELDER UND -AKTEURE}

Als wichtigste Forschungsschwerpunkte der Volga German Studies lassen sich Genealogie und Biographieforschung (vgl. Auman 2017), Migrationsgeschichte und Kolonisierungsgeschichte des unteren Wolgagebiets in der zweiten Hälfte des 18. Jahrhunderts (vgl. Eisfeld 1999-2008, Eichhorn 2012), Zwangsmigration im Kommunismus, Gewalterfahrung und kollektive Wahrnehmungen im 20. Jahrhundert (vgl. Dönninghaus et al. 2018) nennen. Eine kritische Reflexion des Forschungsstandes zur Geschichte und Kultur der Deutschen aus Russland lieferten Katrin Boeckh (2018) und Anke Hilbrenner (2018). ${ }^{2}$ Im Folgenden werden einige derzeitigen Forschungsfeldern der Volga German Studies (wie Genealogie, Religionsgeschichte, Folklore und Literaturwissenschaft) entstammende Beispiele dargestellt, um auf diese Weise Impulse zur Erweiterung von traditionellen Forschungsperspektiven zu setzen. Die Quellengrundlage bilden weniger bekannte Materialien aus dem Staatlichen Historischen Archiv der Wolgadeutschen.

1 Die Deutschen wanderten zwischen 1763 und 1800 auf Einladung der Kaiserin Katharina II. ins Russische Reich ein und ließen sich im unteren Wolgagebiet - in den Gouvernements Saratov und Samara - nieder. Die Einwanderer (ca. 40.000 Personen) stammten mehrheitlich aus Hessen, Nassau und der Pfalz und entschlossen sich für die Auswanderung vor dem Hintergrund des Siebenjährigen Krieges, der Teuerung und Einschränkung der religiösen Freiheit. Die Kaiserin versprach jedoch finanzielle und materielle Unterstützungen für die Einwanderer und Religions-, Gewerbe- und Steuerfreiheit. Diese Privilegien bildeten den Auftakt zum wirtschaftlichen und kulturellen Aufstieg der Wolgadeutschen im langen 19. Jahrhundert (vgl. German/Pleve 2002).

2 Mit voller Anerkennung der Relevanz etablierter Forschungsschwerpunkte wurde für die Öffnung des Forschungsfeldes für transnationale Geschichte, historische Komparatistik, Sozial-, Global-, Verflechtungs- und Transfergeschichte sowie Gender Studies plädiert (Boeckh 2018: 254-256, Hilbrenner 2018: 29), auf der anderen Seite wurde die Festigung der Opfernarrative durch Geschichtsschreibung, der starke zeithistorische Fokus und die Ereignisorientiertheit der vorhandenen Forschungen kritisiert (Boeckh 2018: 254, Hilbrenner 2018: 35). Die Erweiterung des Forschungsfeldes soll nicht zuletzt durch die Einbeziehung von weniger bekannten Archiv- und Quellenmaterialien und stärkere Zusammenarbeit zwischen einzelnen involvierten Disziplinen und Wissenschaftlerinnen und Wissenschaftlern aus unterschiedlichen Ländern stattfinden (Boeckh 2018: 257). 
Bevor die exemplarischen Beispiele behandelt werden können, soll eine wichtige Frage geklärt werden, und zwar wo und von wem die Volga German Studies betrieben werden. Hierbei kann man unter Forschungsinstituten, Universitäten und NGOs unterscheiden. Einige der größten Forschungszentren sind: der aus Mitteln der deutschen und russischen Regierungen finanzierte „Internationale Verband der Deutschen Kultur" (mit dem jährlichen Gesamtbudget von ca. 20 Mio. Euro) in Moskau, die Stiftung „Wiedergeburt“ in Astana, das „Zentrum der russlanddeutschen Geschichte und Kultur" an der Staatlichen Universität Saratov (Russland), des Weiteren das „Nordost-Institut“ (Lüneburg), das „Bundesinstitut für Kultur und Geschichte der Deutschen im östlichen Europa“ (Oldenburg), das Forschungszentrum „Deutsch im Mittel-, Ost- und Südosteuropa“ (Regensburg), das „Center for Volga German Studies“ an der Concordia University (USA), das „Center for Mennonite Studies“ an der University of Winnipeg (Kanada) und die „Pontificia Universidad Católica“ (Buenes Aires, Argentinien). Die geographische Verbreitung der Forschungszentren hängt nicht zuletzt mit vorhandenen Quellenrepositorien zusammen, die, abgesehen von staatlichen Zentralarchiven und zahlreichen Lokalarchiven in diversen Staaten, das „Staatliche Historische Archiv der Wolgadeutschen" in Engels (Russland), die Sammlungen an den Universitäten Concordia, Colorado und Stanford und die „Germans from Russia Heritage Collection“" an der North Dakota State University Library (USA) umfassen. Die einzelnen Archive und Sammlungen unterscheiden sich hinsichtlich des Zuganges und der Nutzungseinschränkungen erheblich, was auch die Episteme der modernen Volga German Studies beeinflusst. Darüber hinaus gibt es auch sprachliche Probleme in Bezug auf unterschiedliche Sprachen der Quellen, einzelne Wissenschaftssprachen wie auch Wissenschaftstraditionen, was die Wissenschaftskommunikation erschwert. ${ }^{3}$

\subsection{Genealogie}

Dank der reichhaltigen Quellenüberlieferung und dem Interesse der Nachkommen der Wolgadeutschen bleibt die Genealogie nach wie vor eine der populärsten Forschungsrichtungen der Volga German Studies, besonders in Russland, Deutschland und nicht zuletzt in den USA. ${ }^{4}$ Als Quellen werden dabei historische Taufbücher und Zensuslisten aus den wolgadeutschen Kolonien (CGASO: F. 52, 53, GASO: F.180, F. 637, GIA NP: F. 162-285, 387-391), aber auch ausführliche Quelleneditionen genutzt (vgl. Eisfeld 1999-2008, Eichhorn 2012). Auch wenn diese Art des Forschens in erster Linie auf Rekonstruktion einzelner Familiengeschichten abzielt, die für die Nachkommen zweifellos einen immensen Stellenwert haben, scheint diese Vorgehensweise für die Geschichtswissenschaft weniger ertragreich zu sein.

3 Ein Beispiel dafür wäre eine völlig falsche inhaltliche Zuordnung einzelner Archivalien im Staatlichen Historischen Archiv der Wolgadeutschen aufgrund sprachlicher Probleme, auf die in weiterer Folge eingegangen wird (s. GIA NP: F. R-1831, op. 1, d. 293). Auch wenn es vereinzelt Zusammenarbeit zwischen diesen Institutionen gibt, wäre es wünschenswert, den internationalen Austausch zu stärken und Forschungsnetzwerke zu entwickeln, in denen die transatlantische Komponente der Erforschung von Kultur und Geschichte der Wolgadeutschen keineswegs außer Acht gelassen werden soll.

4 In den genannten Ländern wurde daraus nicht selten ein florierendes Geschäft. 
Die traditionellen, gut erforschten Quellen der Genealogie finden - abseits der vorhandenen profunden Forschungen zur Einwanderung in das Wolgagebiet im späten 18. Jahrhundert und zahlreichen epochenübergreifenden Ereignisgeschichten der Wolgadeutschen (vgl. German/Pleve 2002) - auch in anderen Forschungskontexten wenig Anwendung. Dennoch wären sie für andere Fragestellungen, beispielsweise die der Sozialgeschichte, von entscheidender Bedeutung. Die bis dato nichtexistierenden exemplarischen und kontrastiven Fallstudien zu Familien- und Haushaltsstrukturen der Wolgadeutschen in den einzelnen Kolonien könnten ein neues Anwendungsfeld für die Quellen der Genealogie sein. Damit einher gehen die Untersuchungen des Heiratsverhaltens der Wolgadeutschen in Bezug auf die sog. Hajnal-Linie, die sich auf eine geringe Anzahl an Publikationen beschränken (s. dazu Gal'perina 2013: 88-93). Die einzelnen Scheidungsanträge und Beichtbücher bieten nämlich relevante Daten zum Familien- und Sexualleben der Wolgadeutschen und können - diese Ansätze sind allerdings nicht ganz neu - viele wertvolle Anregungen über genderspezifische Aspekte der Sozialgeschichte der Wolgadeutschen in der longue durée liefern (GIA NP: F. 162-285, 387-391). Bis dato fanden sie jedoch nur zu wenig Anwendung in den Studien zu Wolgadeutschen, auch wenn sich die Quellenlage für eine solche Art der Untersuchungen anbietet.

Ein weiteres wichtiges Forschungsfeld, zu dem die Quellen der Genealogie beitragen könnten, ist die Verwaltungs- und Verfassungsgeschichte des Russischen Reiches. Seit der Einwanderung und bis zum Zusammenbruch des Zarenreiches hatte die Verwaltung der Wolgadeutschen mehrere unterschiedliche Formationen, von der Selbstverwaltung bis zur vollen Eingliederung in das russländische Rechts- und Verwaltungssystem (vgl. German/Pleve 2002), die nach wie vor einer genaueren Fokussierung bedürfen. Somit wären - neben der Institutionengeschichte - auch die Verwaltungspraktiken und die Praktiken des Dokumentierens auf unterschiedlichen Verwaltungsebenen lohnende Desiderate. Hierbei sollte der Rolle der absichtlichen Fehler der Institutionen und Unklarheiten (Lügen sowie Fehler akustischer Natur etc.) im Verwaltungsprozess ebenfalls Aufmerksamkeit geschenkt werden. Damit könnte nicht nur die Beschaffenheit und Funktion dieser Fehler erklärt werden, sondern auch die daraus resultierenden Konsequenzen für die weitere Auswanderung der Wolgadeutschen, die ihren Niederschlag sowohl in der Vergangenheit als auch in der Gegenwart finden. Die Begegnungen der Wolgadeutschen mit der staatlichen Bürokratie auf unterschiedlichen Ebenen könnten auf der Grundlage der genealogischen Quellen systematisch erforscht werden.

\subsection{Religionsgeschichte}

Vereinzelte Ereignisgeschichten katholischer und evangelischer Kirchengemeinden (vgl. Litzenberger 1995), eingehende Forschungen zu Pietismus und Mennoniten (Evseev 2016) sowie Biographien von wolgadeutschen kirchlichen Amtsträgern (vgl. Barton 1969, Finze-Michaelsen 1992) nutzen als Quellen Kirchenchroniken, Verwaltungsdokumente und Schriften der wolgadeutschen Geistlichen (vgl. GIA NP: F. R-1831, op. 1, dd. 59, 70, 78, 79, 290, 295). Jedoch bleiben in der Beschäftigung mit Volga German Studies viele Forschungsstränge der modernen Religionsgeschichte ausgeblendet, wie etwa die Forschungen zum Glauben und Aberglauben im unteren Wolgagebiet. 
Die unveröffentlichten Archivquellen bieten in Form von Eingaben und Klagen der Wolgadeutschen, Berichten der katholischen und evangelischen Pfarrer über die Sekten sowie Gutachten der höheren kirchlichen Amtsträger eine solide Quellenbasis für die Untersuchungen des religiösen Lebens der Wolgadeutschen, und zwar abseits der kanonischen Formen der Religiosität (GIA NP: F. R-1831, op. 1, dd. 87, 301).

Es ist nicht von der Hand zu weisen, dass in das untere Wolgagebiet über mehrere Jahrzehnte hinweg Schweizer reformierte Pfarrer einwanderten, um das kirchliche Leben in den wolgadeutschen Kolonien aufrechtzuerhalten (vgl. Finze-Michaelsen 1992). Das lässt auf Netzwerke evangelischer Pfarrer in Europa schließen, die nicht nur in die Schweiz (wie im Fall Johann Baptist Cattaneo, Johannes Janett und Hartmann von Moos), sondern auch nach Deutschland und in seltenen Fällen nach Österreich (Ignaz Aurel Feßler) führten. Darüber hinaus begannen sich - nach der Auswanderung mehrerer wolgadeutscher Familien nach Nord- und Südamerika seit den 1870er Jahren (GASO: F. 1) - transatlantische Pfarrernetzwerke zu bilden, die sich in der archivalischen Überlieferung niederschlugen (GIA NP: F. R-1831, op.1, d. 293). Diese vorläufige und nur sehr schematische Bestandsaufnahme ermöglicht, von der Sinnhaftigkeit der Anwendung der sozialen Netzwerkanalyse zur Erforschung der transnationalen Pfarrernetzwerke zu sprechen, an denen sich wolgadeutsche, vorerst evangelische Pfarrer beteiligten. Die religiösen Kontakte zu Nord- und Südamerika trugen erheblich zum Transfer des theologischen Wissens bei. Ein exemplarisches Beispiel dafür stellt der Briefwechsel des amerikanischen evangelisch-lutherischen Pfarrers John Müller aus Altoona (Pennsylvania) mit dem wolgadeutschen Pfarrer Johannes Erbes dar. In dem in Kurrentschrift geführten Briefwechsel geht es um die Zusendung der wolgadeutschen Kirchenbücher nach Amerika (und nicht der historischen Chroniken, wie von den Archivmitarbeiterinnen und -mitarbeitern fälschlicherweise interpretiert), da es viele deutsche Einwanderer aus dem Wolgagebiet gab (GIA NP: F. 1831, op. 1, d. 293). Solche Quellen ermöglichen nicht nur die Erforschung wechselseitiger Transferprozesse und transatlantischer Verflechtungen, sondern auch des Fortlebens und der Nachwirkungen des wolgadeutschen Kulturgutes in anderen historischen und sozio-kulturellen Kontexten.

\subsection{Liedgut und Folklore}

Zur Erforschung und Darstellung des wolgadeutschen Kulturgutes bietet sich das zwischen den 1820er Jahren und 1941 42mal aufgelegte „Wolgagesangbuch“ an als eines der wichtigsten evangelisch-lutherischen Gesangbücher im vorrevolutionären Russland, das nicht nur in Nord-, sondern auch in Südamerika eine breite Anwendung fand (Hildt 2013: 10). Die kulturellen Kontexte des Zustandekommens, der Weiterentwicklung und Adaptation des „Wolgagesangbuches“ an aktuelle Bedürfnisse der Wolgadeutschen im Zarenreich und im Ausland über mehr als hundert Jahre hinweg sind aus kulturhistorischer Sicht eine genauere philologisch-historische Untersuchung wert (vgl. Wolgagesangbuch 1940). Das Gesangbuch könnte auch unter dem musikwissenschaftlichen Aspekt erforscht werden, da es vom Transfer des pietistischen Liedguts in der Welt zeugt.

Die geistlichen Lieder der Wolgadeutschen stehen in enger Beziehung zur wolgadeutschen Folklore, die ein völlig unterbelichtetes Forschungsfeld darstellt. Die jüngsten 
Arbeiten haben einen dezidiert zeithistorischen Fokus und beschäftigen sich mit Überbleibseln des wolgadeutschen Volksliedguts heute (vgl. Šiškina 2011, Windholz 2011), während es keine historiographischen Studien über die Folklore der Wolgadeutschen gibt. Die zahlreichen Quellen zur wolgadeutschen Folklore stellen somit ein marginalisiertes Quellenkorpus dar. Dazu gehören die Liederaufzeichnungen aus den Expeditionen von Viktor Schirmunski, die zurzeit im „Deutschen Volksliederarchiv Leningrad“ (Sammlung Schirmunski) im „Archiv der Russischen Akademie der Wissenschaften“ in St. Petersburg aufbewahrt werden, sowie die Dokumentation der ethnographischen Erkundungen des unteren Wolgagebiets von Clara Obert, August Lohnsinger, Georg und Emma Dinges, die im „Staatlichen Historischen Archiv der Wolgadeutschen“ in Engels

vorliegen (GIA NP: F. R-1831, op.1, d. 131, 132, 134-142). Elena Šiškina gebrauchte viele von diesen Materialien in ihrer Habilitationsschrift nur zum illustrativen Zweck, um die ,historische Kontinuität" der wolgadeutschen Folklore zu verdeutlichen (Šiškina 2011). Die erwähnten Sammlungen der wolgadeutschen Folklore entstanden vor allem während der 1920er Jahre, als das gezielte Sammeln alles Deutschen in der ASSRdWD zum nationalen Eliteprojekt wurde. Die ethnographischen Expeditionen wurden von führenden Spezialisten auf dem Gebiet der Ethnographie (Lohnsinger) und Sprachwissenschaft (Schirmunski, Ehepaar Dinges und Clara Obert als eine der Schülerinnen von Georg Dinges) unter Einbeziehung der Musikologen (Gottfried Schmieder) durchgeführt. Die meisten gesammelten Lieder wurden phonetisch transkribiert mit von vornherein klar festgelegten Notationsregeln. Jede schriftliche Aufzeichnung enthält Notenmaterial als Beilage und Informationen darüber, von wem, wann und wo das Lied sowohl aufgezeichnet als auch aufgeführt wurde (GIA NP: F. R-1831, op. 1, d. 134-142). Auch wenn sich aus der Expedition von Dinges eine Publikation von ca. 50 Liedern ergab (Dinges 1932), stellen sie nicht einmal fünf Prozent der ganzen öffentlich zugänglichen Überlieferung dar. Die akribische Arbeitsweise der Feldforscherinnen und -forscher und der hervorragende Zustand der archivalischen Überlieferung der wolgadeutschen Volkslieder bieten viele Forschungsmöglichkeiten, die sich von Sprach- und Literaturwissenschaft bis hin zur Ethnomusikologie erstrecken, das gesammelte Liedgut wäre ertragreiches Quellenmaterial auch für dialektologische Studien. Genauso würden Studien zur Entstehungsgeschichte einzelner Volkslieder die kulturellen Kontexte der Entstehung eines spezifischen wolgadeutschen Volksliedguts verdeutlichen.

\subsection{Literatur}

Die Folklore geht Hand in Hand mit der Literatur der Wolgadeutschen, die zurzeit als „,zurückgewonnene“ Literatur fungiert, da die Wolgadeutschen nach 1941 aus dem literarischen Leben der Sowjetunion ausgeschlossen wurden (Seifert 2012). Die vor kurzem publizierten Anthologien der russlanddeutschen Literatur, die als eine Art Kanonbildung aufgefasst werden können, haben ihren Fokus auf Gegenwartsautoren, die das russlanddeutsche Opfernarrativ repräsentieren (Seifert 2012), sodass viele Autorinnen und Autoren, die vor 1941 wirkten, unsichtbar und in der Forschung kaum bekannt sind. Darüber hinaus ist die sprachliche Abgrenzbarkeit der wolgadeutschen Gegenwartsliteratur problematisch, da sie auf Deutsch, Russisch und in der wolgadeutschen 
Mundart verfasst wird (Seifert 2012: 19). Dabei wird außer Acht gelassen, dass die Nachkommen der Wolgadeutschen ihre Werke ebenfalls auf Englisch und Spanisch schreiben (vgl. Weidenweber 2008). Somit gibt es nach wie vor keine Literaturgeschichte der Wolgadeutschen seit dem 18. Jahrhundert bis zur Gegenwart, obwohl dies ein dringendes Forschungsdesiderat wäre. ${ }^{5}$

Unter bestimmten Voraussetzungen kann die Literatur auch als historische Quelle fungieren. Das trifft auf die autobiographischen Erzählungen von Alexander Peterson zu, in denen der Autor die Ereignisse des Bürgerkrieges (1917-1923) und Durchsetzung der Sowjetmacht im unteren Wolgagebiet kritisch beschrieb (GIA NP: F. R-1831, op. 1, d. 157). Diese literarisierten Erinnerungen ergänzen die Repräsentation des Bürgerkrieges im Volksliedgut (GIA NP: R-1831, op. 1, d. 136, 25f.) und in der historiographischen Forschung (Jaščenko 2008). Exemplarisch geht es um den Massenterror des roten Kommandeurs Kirill Vakulin, der für viele Verwüstungen im unteren Wolgagebiet verantwortlich war (Jaščenko 2008). Die Erinnerung an diese Tragödie findet sich in der wolgadeutschen Folklore. So enthält ein Lied aus der Kolonie Blumenfeld, das über die Gräueltaten während des russischen Bürgerkrieges erzählt, folgende Versen: „Ich möchte Wakulin nicht sehen, // Hätt ich meine Glieder noch“ (GIA NP: R-1831, op. 1, d. 136, 25f.). Dieselbe emotionelle Aufladung haben auch Werke von Peterson. Das macht deutlich, dass die Einbeziehung weniger bekannter Archivquellen zur Geschichte und Kultur der Wolgadeutschen zu unterschiedlichen Themen, darunter auch zur Erforschung des russischen Bürgerkrieges, einen nicht unwesentlichen Beitrag zu den Volga German Studies leisten kann.

\section{SCHLUSS}

Zusammenfassend lässt sich festhalten, dass die Volga German Studies ein Forschungsfeld darstellen, in dem Forschungsfragen zur Funktion der Genealogie, der Religionsgeschichte abseits der kanonischen Formen, der geistlichen Lieder, des Wolga-Volkliedes und der Folklore wie auch der Bedeutung der wolgadeutschen Literatur unter verschiedenen disziplinenrelevanten Aspekten künftig noch beantwortet werden müssen. Die hier skizzierten Möglichkeiten für die interdisziplinäre Forschung konnten nicht die ganze Palette möglicher Ansätze und Forschungsdesiderate abdecken, die noch ihrer wissenschaftlichen Aufarbeitung harren. Der kleine Überblick konnte allerdings verdeutlichen, dass die Gründung neuer interdisziplinärer Forschungsgruppen und Beantragung von innovativen Projekten zur Geschichte und Kultur der Wolgadeutschen vom 18. Jahrhundert bis heute notwendig ist. Es wurde gezeigt, dass dazu auch die unveröffentlichten Archivquellen maßgebend beitragen können. Nicht zuletzt hängt die Zukunftsträchtigkeit der Volga German Studies und ihre Umwandlung zu einem innovativen Forschungsfeld von

5 Auch der wolgadeutschen Kinder- und Jugendliteratur wird eine hohe gesellschaftliche Relevanz zugesprochen. Als Vertreterin wird meistens die Angehörige der Minderheit, Schriftstellerin Clara Obert genannt. Trotz guter Quellenüberlieferung im Archiv gibt es keinerlei Editionen, die ihr CEuvre für die interessierte Öffentlichkeit zugänglich gemacht hätten (GIA NP: F. R-1831, op. 1, d. 144). Dasselbe gilt für weitere wolgadeutsche Autoren wie Winzo Klang, Waldemar Herdt, Victor Klein, Ferdinand Walberg und viele andere (GIA NP: F. R-1831, op. 1, dd. 149, 151, 156, 207). 
Handlungen der Wissenschaftlerinnen und Wissenschaftler ab, die sich mit diesem komplexen und dynamischen Forschungsfeld beschäftigen.

\section{Literatur}

AUMAN, Vladimir (2017) Gerold Belger. Takaja vypala stezja. Izvestnye nemcy Kazachstana. Almaty: Vozroždenie.

BARTON, Peter (1969) Ignatius Aurelius Feßler. Vom Barockkatholizismus zur Erweckungsbewegung. Wien: Böhlau.

BOECKH, Katrin (2018) „Stand und Perspektiven der Historiographie zu den Russlanddeutschen." In: V. Dönninghaus/J. Panagiotidis/H.-Ch. Petersen (Hrsg.), 251263.

Central'nyj gosudarstvennyj archiv Samarskoj oblasti (CGASO). F. 52, 53.

DINGES, Georg (1932) Wolgadeutsche Volkslieder mit Bildern und Weisen. Berlin: De Gruyter.

DÖNNINGHAUS, Victor/Jannis PANAGIOTIDIS/Hans-Christian PETERSEN (Hrsg.) (2018) Jenseits der „Volksgruppe“. Neue Perspektiven auf die Russlanddeutschen zwischen Russland, Deutschland und Amerika. Oldenburg: De Gruyter.

EICHHORN, Alexander, Jacob und Maria (Hrsg.) (2012) Die Einwanderung deutscher Kolonisten nach Dänemark und deren weitere Auswanderung nach Russland in den Jahren 1759-1766. Bonn: Selbstverlag.

EISFELD, Alfred (Hrsg.) (1999-2008) Einwanderung in das Wolgagebiet. 1764-1767. Göttingen: Göttinger Arbeitskreis.

EVSEEV, Nikolaj (2016) Mennonity v saratovskom Zavolž’je. Saratov.

FINZE-MICHAELSEN, Holger (1992) Von Graubünden an die Wolga: Das Leben des Bündner Pfarrers Johannes Baptista Cattaneo (1745-1831) und seine Zeit. Chur: Bündner Monatsblatt.

GAL'PERINA, Julija (2013) „Semejnaja struktura nemeckich poselenij na Volge v seredine XIX v.: opyt issledovanija kolonii Gniluška (Pfajfer). “ Izvestija Saratovskogo gosudarstvennogo universiteta. Istorija. Meždunarodnye otnošenija, 13/4, 88-93.

GERMAN, Arkadij/Igor PLEVE (2002) Nemcy Povolžja. Kratkij istoričeskij očerk. Saratov: Izdatel'stvo Saratovskogo gosudarstvennogo universiteta.

Gosudarstvennyj archiv Saratovskoj oblasti (GASO). F. 1, 180, 637.

Gosudarstvennyj istoričeskij archiv nemcev Povolž'ja (GIA NP). F. 162-285, 387391. F. R-1831, op. 1, dd. 59, 70, 78, 79, 87, 131, 132, 134-142, 144, 149, 151, 156, 157, 207, 290, 293, 295, 301.

HILBRENNER, Anke (2018) „Russlanddeutsche und andere Zugehörigkeiten: Der Begriff der „Identität“ zwischen Erinnerung und Geschichte.“ In: V. Dönninghaus/J. Panagiotidis/H.-Ch. Petersen (Hrsg.), 29-37.

HILDT, Leandro (2013) „Libros rescatados: Wolgagesangbuch.“ Vida abundante, $118 / 2,10$.

JAŠČENKO, Vjačeslav (2008) Antibol'ševistskoe povstančestvo v nižnem Povolž'e i na srednem Donu. 1918-1923. Volgograd: Liberkom. 
KAPPELER, Andreas (Hrsg.) (1996) Regionalismus und Nationalismus in Rußland. Baden-Baden: Nomos.

KLAUS, Alexander (2010) Unsere Kolonien: Studien und Materialien zur Geschichte und Statistik der ausländischen Kolonisation in Russland. Hildesheim: Georg Olms.

LITZENBERGER, Olga (1995) Evangeličesko-ljuteranskaja cerkov' Sv. Marii v g. Saratove (1770-1935). Saratov: Izdatel'stvo Saratovskogo gosudarstvennogo universiteta.

SCHMALTZ, Eric (2018) „What's in a Name? Russian Germans, German Russians, or Germans from Russia, and the Challenges of Hybrid Identities." In: V. Dönninghaus/J. Panagiotidis/H.-Ch. Petersen (Hrsg.), 41-72.

SEIFERT, Elena (Hrsg.) (2012) Der misstrauischen Sonne entgegen. Anthologie der Literatur der Russlanddeutschen der zweiten Hälfte des 20. Jahrhunderts - Anfang des 21. Jahrhunderts. Moskau: IVDK Press.

SMIRNOVA, Tatiana (1992) „Siberian Germans.“ In: Encyclopedia of World Cultures. Vol. 6, Russia and Eurasia/China. Boston: Macmillan Reference, 337-341.

ŠIŠKINA, Elena (2011) Tradicionnoe muzykal'noe nasledie volžskich nemcev v prošlom i sovremennosti. Saratov.

WEIDENWEBER, Sigrid (2008) The Volga Germans. Portland: Center for Volga German Studies at Concordia University.

WINDHOLZ, Johannes (2011) Nemcy Rossii. Pesnja - muzyka - obrjad. Saratov: Novyj veter.

Wolgagesangbuch (1940). Sammlung christlicher Lieder für die öffentliche und häusliche Andacht. Chicago: Wolga Book Company.

\section{Zusammenfassung \\ DIE WOLGADEUTSCHE KULTUR UND LITERATUR - FORSCHUNGSFELDER, -PROBLEME UND -DESIDERATA}

Der vorliegende Artikel bietet einen Überblick über aktuelle Tendenzen der Volga German Studies, die ein komplexes heterogenes Forschungsfeld an der Schnittstelle zwischen Geschichte, Theologie, Philologie und Sozialwissenschaften darstellen. Ausgehend von vorhandenen Zugängen zur Geschichte und Kultur der Wolgadeutschen werden Forschungslücken identifiziert und kritisch diskutiert, wobei neue Forschungsfragen auf Grundlage der zugänglichen Archivquellen entwickelt werden. Die in der Forschungsliteratur unterbelichteten Archivquellen werden vorgestellt sowie ihr Wert für interdisziplinäre Forschungen zu den Wolgadeutschen fokussiert. Besondere Aufmerksamkeit wird auf den Wert von älteren genealogischen, religionsgeschichtlichen und literaturwissenschaftlichen Forschungen für neue interdisziplinäre Fragestellungen gerichtet.

Schlüsselwörter: Volga German Studies, Interdisziplinarität, Religionsgeschichte, Literatur, Archiv der Wolgadeutschen. 


\section{Abstract \\ VOLGA GERMAN LITERATURE AND CULTURE - RESEARCH FIELDS, PROBLEMS AND DESIDERATA}

This article offers an overview of recent trends in and perspectives of Volga German Studies, which is understood as a complex and heterogenous research field, bringing together history, theology, philology and the social sciences. After presenting the most recent and current approaches, research gaps are then discussed critically, and new research questions are developed light of existing archival sources. Sources which have not thoroughly been incorporated into historical research are presented, and their value for interdisciplinary research on Volga Germans is highlighted. Particular emphasis is placed on the value of previous research for the proposed new interdisciplinary approaches, especially in the fields of genealogy as well as the history of the religion, folklore and literature of the Volga Germans.

Keywords: Volga German Studies, interdisciplinarity, history of religion, literature, Volga German archives.

\section{Povzetek \\ KULTURA IN KNJIŽEVNOST POVOLŠKIH NEMCEV - RAZISKOVALNA PODROČJA, PROBLEMI IN DEZIDERATI}

$\mathrm{V}$ članku je predstavljen pregled aktualnih tendenc v okviru raziskovanja Nemcev na Volgi (Volga German Studies). Raziskovanje manjšine povolških Nemcev predstavlja kompleksno in heterogeno raziskovalno področje, ki se nahaja med zgodovino, teologijo, filologijo in družbenimi vedami. Izhajajoč iz obstoječih pristopov k zgodovini in kulturi povolških Nemcev je cilj prispevka identificirati in kritično ovrednotiti raziskovalne vrzeli, ob tem pa na osnovi obstoječih arhivskih virov pokazati na nove raziskovalne perspektive. V prispevku so predstavljeni arhivski viri, ki so $\mathrm{v}$ znanstveni literaturi na temo povolških Nemcev premalo osvetljeni. Z namenom nadaljnjega meddisciplinarnega raziskovanja povolške nemške manjšine je posebna pozornost posvečena pomenu starejših genealoških, religijskozgodovinskih in literarnovednih raziskav.

Ključne besede: študije povolških Nemcev, meddisciplinarnost, zgodovina religije, književnost, arhiv povolških Nemcev 